\title{
A new methodology to identify minimum strain anatomicall lines based on 3-D digital image correlation
}

\author{
Jorge Barrios-Muriel, Francisco Javier Alonso Sánchez, David Rodríguez Salgado, and \\ Francisco Romero-Sánchez \\ Department of Mechanical Engineering, Energy and Materials, University of Extremadura, \\ Avda. de Elvas s/n, 06006, Badajoz, Spain \\ Correspondence to: Jorge Barrios-Muriel (jorgebarrios@unex.es)
}

Received: 14 April 2017 - Revised: 5 October 2017 - Accepted: 19 October 2017 - Published: 16 November 2017

\begin{abstract}
Today there is continuous development of wearable devices in various fields such as sportswear, orthotics and personal gadgets, among others. The design of these devices involves the human body as a support environment. Based on this premise, the development of wearable devices requires an improved understanding of the skin strain field of the body segment during human motion. This paper presents a methodology based on a three dimensional digital image correlation (3D-DIC) system to measure the skin strain field and to estimate anatomical lines with minimum deformation as design criteria for the aforementioned wearable devices. The errors of displacement and strain measurement related to 3-D reconstruction and out-of-plane motion are investigated and the results are acceptable in the case of large deformation. This approach can be an effective tool to improve the design of wearable devices in the clinical orthopaedics and ergonomics fields, where comfort plays a key role in supporting the rehabilitation process.
\end{abstract}

\section{Introduction}

Understanding of the skin's properties during human motion is critical in the design of any device that is in contact with the human body. Muscle contractions cause displacements in the soft tissues and deformations of the skin that eventually cause chafing or bedsores and general discomfort at the human-device interface. Thus, skin strain analysis may provide an insight into the design of devices where the mechanical loads on the skin must result in comfort and safety. These loads produce tangential and normal strain that can cause skin injuries. Normal strain to the skin is related to ulcers and bedsores and tangential strain to wounds and chafing (Vilhena and Ramalho, 2016; Kwiatkowska et al., 2009). The latter are the most common cases. Thus, only tangential strain related to these injuries is analysed here. A new design trend has emerged around the idea that the products in contact the human body should be on minimum strain anatomical lines, namely Lines of Non-Extension (LoNEs). This approach based on skin strain analysis was first proposed in the work of Iberall (1964) and was continued by Newman et al. (2005) to improve the mobility of astronauts. Today, this methodology is used in a variety of applications, including the design of a prosthetic socket (Lin et al., 2016), orthoses (Domingues et al., 2011), exoskeletons for gait assistance (Wehner et al., 2013) and functional clothes (Seo et al., 2013), among others.

From a mechanical point of view, human skin presents different patterns of deformation mainly related to its anisotropic and elastic properties (Geerligs et al., 2011a, b). Previous studies have focused on the measurement of the material properties of isolated skin samples by means of in vitro tensile tests (Geerligs et al., 2011a, b; Annaidh et al., 2012a, b). However, these studies have a limitation in the fact that the skin behaves differently when it is removed from surrounding tissues. Unlike the in vitro tensile test, in vivo examination of skin allows the measurement of its mechanical properties in a natural state of pre-tension and hydration. The anisotropy of human skin varies from one anatomical segment of the body to another (Annaidh et al., 2012a, b). As a result of the mechanical complexities in modeling skin, empirical skin strain data is particularly relevant to understand skin's behaviour during human motion. 


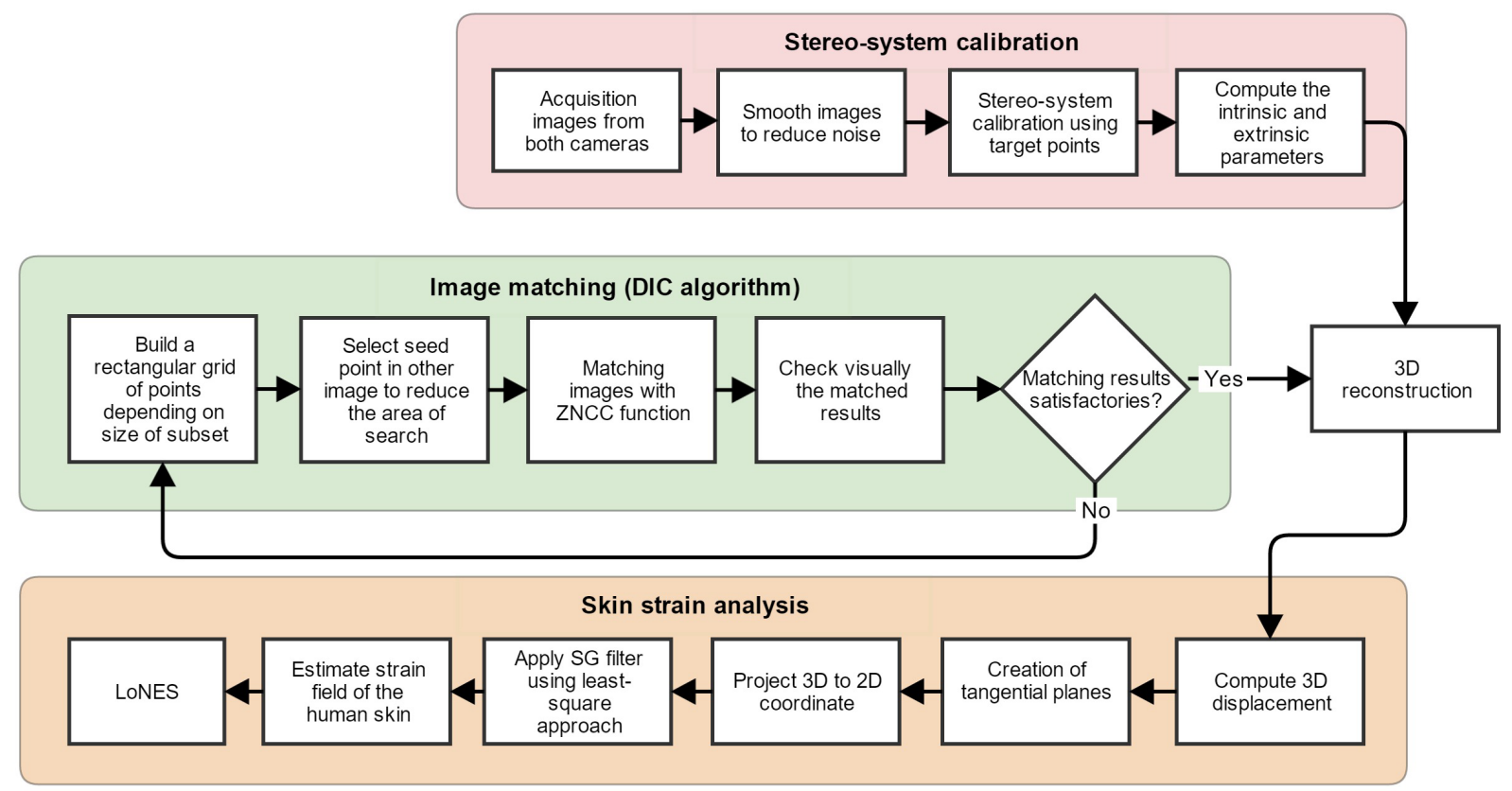

Figure 1. Flowchart of the methodology of 3D-DIC algorithm and skin strain analysis.

At present, there is no standard procedure for skin strain analysis. Different techniques and technologies have been used to this end: magnetic resonance imaging (Tran et al., 2007), optical coherence tomography (Hendriks et al., 2006), ultrasound (Hendriks et al., 2003), 3-D scanning (Van den Herrewegen et al., 2014), multiple-view stereo matching (Thabet et al., 2014; Kimura et al., 2008), motion capture systems (Mahmud et al., 2010) and digital image correlation (DIC) (Staloff et al., 2008; Evans and Holt, 2009; Miura et al., 2012; Yoneyama, 2010; Obropta and Newman, 2016). At the same time, the procedure to calculate skin strain depends on the technology used. The most commonly used procedures for this purpose combine optical techniques with finite strain theory and the strain gauge rosette method (Obropta Jr., 2015; Evans and Holt, 2009; Miura et al., 2012; Staloff et al., 2008). The finite strain theory combined with 3-D digital image correlation (3D-DIC) has been shown to be the most accurate method as it provides the highest spatial resolution to measure the skin strain field during movement (Miura et al., 2012; Obropta Jr., 2015; Staloff et al., 2008). Commercial DIC systems have been used successfully to analyze 3-D skin strain (Moerman et al., 2009; Obropta and Newman, 2016). However, DIC systems are expensive and unaffordable in many cases. The objective of this work is to present a low-cost methodology to perform skin strain analysis using the 3D-DIC approach. In the following sections we present the proposed methodology and a series of validation tests performed on different objects. Then the methodology is applied to the human body, specifically to the ankle joint complex. To this end, speckle patterns were applied to the skin, and the principles of image correlation and stereo trian- gulation were used to measure 3-D deformation of the body segment. The skin strain field was then calculated to obtain the anatomical lines with minimum strain (LoNEs) using the finite strain theory. Based on these LoNEs, the basic structure of the wearable device was optimized in order to improve comfort and usability.

\section{Methodology}

DIC is a non-contact optical tool for surface deformation measurement that has been widely accepted and used in the field of experimental mechanics (Chu et al., 1985; Réthoré et al., 2007; Elguedj et al., 2011; Gu et al., 2016). The main advantages of this methodology are the simplicity of the data acquisition devices, the ease of measurement and also its application in large deformation cases. In essence, DIC is a technique based on digital image processing and numerical computing whose basic principle is to track the same physical points located in the undeformed and deformed images. In 3D-DIC (also called stereo-DIC), the 3-D surface object is reconstructed by processing the images captured by two cameras with different perspectives. A random gray-value dot pattern is painted on the surface (in this case on the human skin) to perform a correspondence between points of the two images using the DIC algorithm. A comprehensive description of the metrological aspects can be found in (Luo et al., 1993; Tang et al., 2012). Three primary steps are differentiated in the proposed methodology: stereo-system calibration, image recording and matching and, finally, 3-D reconstruction and computation of the strain field (Fig. 1). 


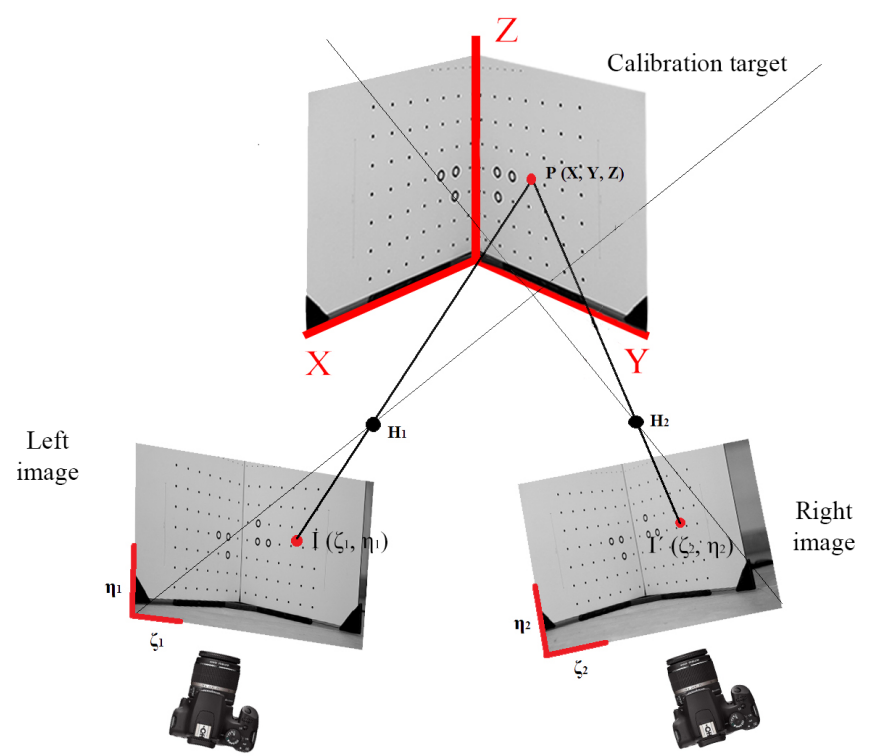

Figure 2. Scheme of the calibration process using an object with know control points.

\subsection{Stereo-system calibration}

Camera calibration is a necessary step in order to create a metric relation between the captured 2-D images and the 3-D surface. The main goal of this process consists in determining the intrinsic and extrinsic parameters of each camera. On the one hand, the geometric properties of the camera such as focal length, principal axis and sensor size are related to the intrinsic parameters and on the other hand, the relative positions or/and orientation of the camera with respect to a world coordinate system are related to the extrinsic parameters. Different methods to calibrate stereo systems have been presented in the literature, but the Direct Linear Transformation (DLT) is the basis of most of the reported calibration techniques. Its principle is to calculate the intrinsic and extrinsic parameters from a known object with control points (calibration target).

In this work we adopt the DLT algorithm (Abdel-Aziz and Karara, 1971) modified by Hatze (1988) and implemented in MatLab ${ }^{\circledR}$ by Genovese et al. (2011). This method is based on the pinhole model camera assumption, where the camera aperture is described as a point and no lenses are used to focus light. To make the transformation from sensor coordinates to world coordinates we apply a rigid body transformation where the vector of interest is multiplied by a rotation matrix (intrinsic parameters) and added a translation vector (extrinsic parameters) as follows:

$$
\begin{aligned}
& \zeta_{1}-\zeta_{2}=l \cdot s_{\zeta} \frac{\left(M_{x x} X+M_{x y} Y+M_{x z} Z+\delta_{x}\right)}{\left(M_{z x} X+M_{z y} Y+M_{z z} Z+\delta_{z}\right)} \\
& \eta_{1}-\eta_{2}=l \cdot s_{\eta} \frac{\left(M_{y x} X+M_{y y} Y+M_{y z} Z+\delta_{y}\right)}{\left(M_{z x} X+M_{z y} Y+M_{z z} Z+\delta_{z}\right)}
\end{aligned}
$$

where $\zeta$ and $\eta$ are the 2-D coordinate point onto camera sensor, $l$ the focal length and $s_{\zeta}, s_{\eta}$, the scale factors that represent ratios between lengths of sensor and the world coordinate systems. $M_{a b}$ and $\delta_{a}(a, b=x, y, z)$ are the three independent components of the matrix rotation and the translation vectors, respectively. $X, Y, Z$ are the point coordinates in the global coordinate system.

In the DLT method the goal is to simplify the solution of both Eqs. (1) and (2) by substituting the unknown parameters with a series of constants called $L_{n}$. The basic method uses 11 parameters, and depends on how many corrections are to be done to the optical system (such as spherical distortion, astigmatism, etc). For more details see Abdel-Aziz and Karara (1971), Genovese et al. (2011). This constants are functions of the extrinsic and intrinsic parameters of each camera (Abdel-Aziz and Karara, 1971), thus the Eqs. (1) and (2) can be rearranged as:

$\zeta=\frac{L_{1} X+L_{2} Y+L_{3} Z+L_{4}}{L_{9} X+L_{10} Y+L_{11} Z+1}$
$\eta=\frac{L_{5} X+L_{6} Y+L_{7} Z+L_{8}}{L_{9} X+L_{10} Y+L_{11} Z+1}$

Each control point provides two equations, so a minimum of $n=6$ control points is necessary to extract the entire set of 11 DLT parameters. However, more control points are used to reduce the experimental errors. In this work 98 control points were used (Fig. 2)

The result of the camera calibration step is a transformation that assigns a 3-D world point $\mathrm{P}(X, Y, Z)$ into a 2-D sensor image point $\mathrm{I}(\zeta, \eta)$ assuming that the points $\mathrm{P}, \mathrm{I}$ and $\mathrm{I}^{\prime}$ and the pinhole $\mathrm{H}$ are collinear (collinearity condition of the DLT method).

\subsection{Image matching}

The main difficulty in 3D-DIC is to establish correspondences between pairs of images from each camera. Different algorithms have been proposed based on contour conditions, but the most used are based on template matching. In this work, the principle of DIC algorithm is based on template matching. Generally, to correlate the two stereo-views, DIC algorithm requires a random speckle pattern to match the corresponding point-pairs seen by both cameras (Fig. 3a). This allows finding the best match between corresponding points in the two images by comparing the local grey scale distribution of square pixels subsets on the basis of the normalized cross correlation coefficient. This process correlates the grey levels of a window fixed in the first image (reference image) with a shifting window in the second (current image) (Fig. 3b). To this aim, the size subset is first defined to create a rectangular grid of points that represent the centres of the subsets of interest and, second, a cross-correlation or a sum of squared differences (SSD) criterion to evaluate the 
(a)

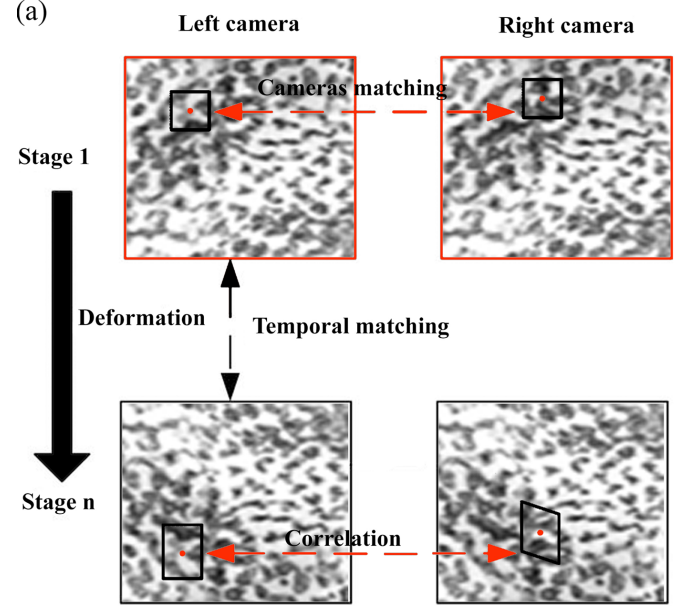

(b)

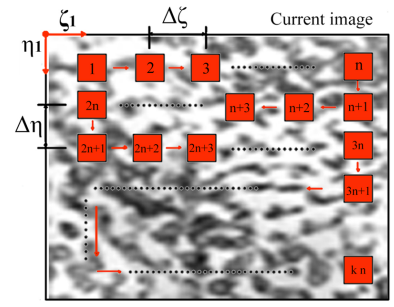

(c)

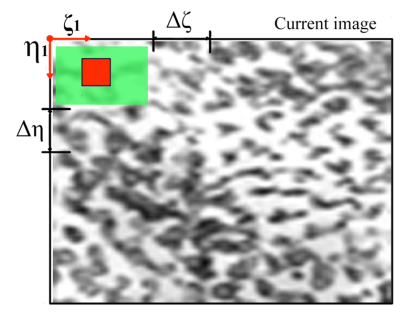

Figure 3. (a) Temporal and cameras match process. (b) Typical path performed by each reference subset during the search of matched area. Red squares are the positions of a reference subset by all current image during typical path. (c) seed-point approach to correlate subsets. Green squared is the reduced area of search. $\Delta \zeta$ and $\Delta \eta$ are the distance between centre of subsets and $\zeta_{1}, \eta_{1}$ the sensor coordinate system.

similarity between subsets is used. The zero-mean normalized correlation criterion (ZNCC) has been strongly recommended for practical use since it is robust against changes in image's scale and intensity offset (Pan et al., 2007, 2010). ZNCC is optimized using a Newton-Raphson (NR) method to calculate the displacement at each point.

$$
\Psi=\frac{\sum_{a}^{b} \sum_{a}^{b}[f(\zeta, \eta)-\bar{f}]\left[g\left(\zeta^{\prime}, \eta^{\prime}\right)-\bar{g}\right]}{\sqrt{\sum_{a}^{b} \sum_{a}^{b}[f(\zeta, \eta)-\bar{f}]^{2}} \sqrt{\sum_{a}^{b} \sum_{a}^{b}\left[g\left(\zeta^{\prime}, \eta^{\prime}\right)-\bar{g}\right]^{2}}}
$$

where $\Psi$ is the ZNCC, $f(\zeta, \eta)$ and $g\left(\zeta^{\prime}, \eta^{\prime}\right)$ are the greyscale values at the pixel $(\zeta, \eta)$ in the reference and shift window, respectively. $\bar{f}$ is the mean value of $f(\zeta, \eta)$ over the reference subset of dimension $a \times b$ pixels and $\bar{g}$ is the mean value of $g\left(\zeta^{\prime}, \eta^{\prime}\right)$ over the subset of current image. The maximum value of $\Psi$ is the best match between two images.

In this work, to optimize the computation time of the correlation function, a similar seed-point approach was used (Pan and Li, 2011) which allowed for the eliminating of the repeating redundant calculations involved in the correlation process. An initial value for the centre of the first subset reference is chosen manually in the current image in order to reduce the search area of reference subset in all images. The displacement obtained is added to the next search area (green squared in Fig. 3c) of the next reference subset and thus it moves together with the next reference subsets. The size of square subset in our analysis was 40 pixels. Moreover, an unsharp masking was applied to each image to eliminate the blur and improve the functioning of the correlation function.

\subsection{3-D reconstruction}

After the matching process for each stage is carried out, the 2-D matched points in each image can be converted into 3-D coordinates by applying the DLT method. Thus, the calibration parameters of the stereo cameras and the corresponding 2-D matched points in the left and right images are substituted on Eqs. (3) and (4). By solving both equations using a linear least-squares approach, one can obtain the unknown position of each point with respect to the global coordinate system. By repeating this operation for each stereo matched point, the 3-D shape of the surface can be obtained.

\subsection{Skin strain computation}

As a result of the 3-D reconstruction step, a set of physical points in terms of Cartesian coordinates $X^{i}$ with respect to a global reference system are available. 3-D displacement of any point can be calculated directly by comparing its 3-D coordinates in the current stage and the reference stage. To obtain the strain field on a three-dimensional surface, the displacements should be differentiated along the tangential direction of the surface, in this case, the human skin. Direct numerical differentiation is not recommended due to its instability and the amplification of noise. Moreover, in our case, the direction of differentiation of the global coordinate system does not coincide with the tangential planes of the skin and thus it is necessary to carry out a coordinate transformation on each subset of skin analysed in DIC. Generally, the NR method is used in DIC to obtain displacement gradients, but this method cannot adequately estimate the strain field due to the unavoidable influences of different types of noises (Quan et al., 2008). In this sense, it has been found that Savitzy-Golay (SG) filter is able to improve the strain fields 
compared to NR process (Tang et al., 2010; Quan et al., 2008; Yoneyama, 2011; Begonia et al., 2017). For this reason, an approach proposed by Tang et al. (2010) based a 2-D SG filter differentiator is used in this work in order to obtain the strain field. The main idea of this process is to use the 3-D coordinates of eight neighbour points to calculate the strain of point $a_{i, j}$ and to assume that the underlying displacement field can be fitted with a polynomial representation. With this process the noise in the computed displacement field can be easily removed as the SG filter generally preserves features of the distribution such as relative maxima, minima and width. The derivatives of the displacement field obtained from the SG are computed using the coefficients of the fitted polynomial. The process requires the following steps:

1. Calculate in each stage a tangential plane using the neighbour points of point $a_{i, j}$. Then, the 3-D coordinate of the points are projected onto new tangential plane $\left(A_{p}\right)$ to obtain 2-D points $\left(\chi_{i, j}\right) . A_{p}$ is an arbitrary 2-D coordinate system whose axes $\left(p_{x}, p_{y}\right)$ coincide with tangential directions of the human skin.

2. Apply the SG filter and compute the unknown polynomial coefficients using the least-squares method. The SG filter method is based on a polynomial fit to the data and the derivatives from the polynomial. To estimate the strain using 2-D SG filter we use a polynomial regression (of degree $n$ ) on a distribution of $2 M+1$ by $2 M+1$ points (of width $M$ ) around the each point $\mathrm{O}(0,0)$ to determine the derivatives $\left(d_{x}, d_{y}\right)$ of the displacements with respect to the new 2-D coordinate system $\left(A_{p}\right)$ calculated in the above step. Thus, for $\mathrm{M}=1$, 9 grid points are obtained (the current point $\mathrm{O}$ and eight neighbor points). Mathematically, strains can be represented as:

$$
\begin{aligned}
& \varepsilon_{x x}(i, j)=\frac{\partial u}{\partial x}=F_{n_{x}, n_{y}}^{1,0}(i, j) \\
& \varepsilon_{y y}(i, j)=\frac{\partial v}{\partial y}=F_{n_{x}, n_{y}}^{0,1}(i, j) \\
& \gamma_{x y}(i, j)=\frac{\partial u}{\partial y}+\frac{\partial v}{\partial x}=F_{n_{x}, n_{y}}^{0,1}(i, j)+F_{n_{x}, n_{y}}^{1,0}(i, j)
\end{aligned}
$$

where $u, v$ are the displacement, $F$ is the digital differentiator, $n_{x}$ and $n_{y}$ are the polynomial orders and $i, j$ the evaluated points. When applying the 2-D SG filter to the $p_{x}$-direction the $d_{x}=1$ and $d_{y}=0$ and in $p_{y}$ direction, $d_{x}=0$ and $d_{y}=1$. The 2-D SG digital differentiator can be expressed as:

$$
F_{n_{x}, n_{y}}^{d_{x}, d_{y}}(i, j)=\frac{\sum_{i=-M}^{M} \sum_{j=-M}^{M} h_{i}^{d_{x}, d_{y}} h_{j}^{d_{x}, d_{y}} g_{i, j}}{\Delta x^{d_{x}} \Delta y^{d_{y}}}
$$

where $g_{i, j}$ represents the displacements, $h_{i, j}^{d_{x}, d_{y}}$ the convolution weights for data points $\left(a_{i, j}\right)=\left(-m_{i},-m_{j}\right)$ to $\left(m_{i}, m_{j}\right)$, being $M$ the width of the local subregion of the displacement centered at the current point $(i, j)=$ $(0,0) . \Delta x$ and $\Delta y$ are the distances between each data points.

To avoid large errors at the boundary when the SG digital differentiator is employed, the outliers of the data are excluded. These outliers are produced by the SG digital differentiator in the boundary of the cloud points, providing erroneous information (for a more detailed explanation see Pan et al., 2007). In this work the parameters $n_{x}, n_{y}, m_{i}, m_{j}=2$ are used (2nd polynomial order of $5 \times 5$ grid of data points).

\subsection{Calculation of LoNES}

Based on the previous skin strain analysis, we can identify the LoNEs applying the strain ellipse theory. To help understand the concept of LoNEs, we shall refer to the finite strain ellipse and Mohr's circle. In Fig. 4 the concept of the direction of non-extension is presented. In the ellipse, there are two lines with no extensional strain (LoNEs). These LoNEs only exist if the principal strains have opposite signs, and the physical meaning is that the material is undergoing deformation in one direction and compression in the orthogonal direction. LoNEs can be conceptualized as directions where there is no extensional strain along the two lines formed by the intersection of the unit circle and deformed state. In this case, there are two directions where there is only distortion (change of direction) and no deformation $(\varepsilon=0)$. In the present work, when referring to the directions of LoNES, we shall assume that the skin is a two-dimensional material, and the strain field correspond to tangential component of strain as previously mentioned.

The method used to obtain the directions of the LoNEs begins with the calculation of the principal direction of strain using the polar decomposition theorem. An eigenvector analysis of the local strain field at each data point is performed to obtain the eigenvectors $(\boldsymbol{v})$ and eigenvalues $(\lambda)$ of the strain tensor. The LoNES's directions can be calculated by the following expression:

$\theta_{i j}=\arctan \left(\sqrt{-\frac{\lambda_{1 i j}}{\lambda_{2 i j}}}\right)$

where $\lambda_{1 i j}$ and $\lambda_{2 i j}$ are the principal strains magnitude. At this step, we have LoNEs in each area of skin where there has been an extension and compression at a time, but this information is not enough to conclude the process. To avoid the excessive distorsion that there may be in determinate areas, we need to choose the areas with minimum or null distorsion. To do so, we calculate the angle between the directions of non-extension $\left(\phi_{i j}\right)$ as: 


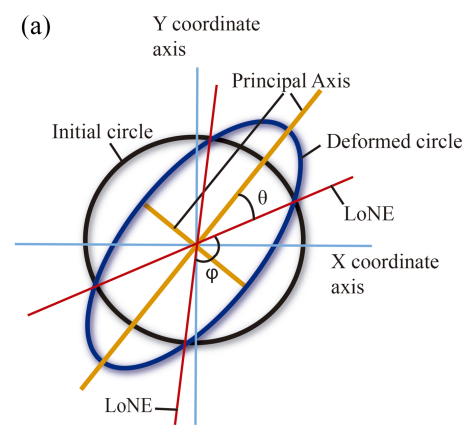

(b)

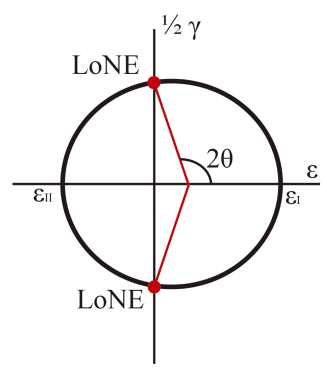

Figure 4. Conceptual representation of LoNES with finite strain ellipse (a) and Mohr's circle (b). The black circle represents the initial (undeformed) shape, the blue ellipse the deformed shape, and the orange lines the principal strain directions.

$\phi_{i j}=\arccos \left(\frac{w_{1 i j}}{w_{2 i j}}\right)$

where $w_{1 i j}$ and $w_{2 i j}$ are the directions of non-extension and $\phi_{i j}$ the angle between the vectors of these directions. To select the most favourable areas we use the root mean square (RMS) of the whole angle, $\phi$, defined as:

$\mathrm{RMS}=\sqrt{\frac{1}{N} \sum_{i=1}^{N} \phi_{i j}}$

as a criteria $(\mu)$ to determine if the areas is favourable or not. This "filtered" factor is based on the condition that the most favourable areas must have a value $\phi-90^{\circ}$ lower to RMS.

$\mu=\left\{\begin{array}{l}\text { if } \phi_{i j}-90^{\circ}<=\text { RMS } \rightarrow \text { Area favorable } \\ \text { if } \phi_{i j}-90^{\circ}>\text { RMS } \rightarrow \text { Area unfavorable }\end{array}\right.$

By using this factor, we ensure the selection of areas with minimal distorsion.

\section{Experimental test to validate methodology}

DIC measurements and skin strain analysis described herein have not been reported previously. Hence, a series of validation tests were performed to assess overall accuracy of the measurement using this methodology. The system can offer many advantages in biomechanics and rehabilitation engineering fields as it allows to gather information over surface of human skin in 3-D in large or small region of interests. Using this system the spatial resolution of measurements is acceptable to large deformation, on the order of $0.5-1 \mathrm{~mm}^{2}$. This is a merit due to the hardware system used in this work consist on two basic DSLR cameras and not uses a highspeed or high resolution camera systems.
Table 1. Errors (mm), mean and SD of 5 samples in each horizontal position (range of displacement) of Test B.

\begin{tabular}{lllllll}
\hline & $10 \mathrm{~mm}$ & $20 \mathrm{~mm}$ & $30 \mathrm{~mm}$ & $40 \mathrm{~mm}$ & $50 \mathrm{~mm}$ & $60 \mathrm{~mm}$ \\
\hline Sample 1 & 0.1213 & 0.0865 & 0.2839 & 0.4207 & 0.3077 & 0.2396 \\
Sample 2 & 0.1932 & 0.1370 & 0.2063 & 0.0535 & 0.1535 & 0.0623 \\
Sample 3 & 0.1121 & 0.1910 & 0.0531 & 0.0531 & 0.0931 & 0.1131 \\
Sample 4 & 0.0957 & 0.0362 & 0.0392 & 0.0962 & 0.0162 & 0.1032 \\
Sample 5 & 0.0832 & 0.0490 & 0.1420 & 0.0790 & 0.0990 & 0.1290 \\
\hline Mean & 0.1211 & 0.0999 & 0.1449 & 0.1405 & 0.1339 & 0.1295 \\
SD & 0.0429 & 0.0642 & 0.1033 & 0.1577 & 0.1088 & 0.0663 \\
\hline
\end{tabular}

\subsection{Test A: error in 3-D reconstruction}

To verify the effectiveness of the 3-D reconstruction of the proposed method, a cylinder with a white and black speckle pattern was used (Fig. 5a). The sample was measured with a caliber and the measured diameter was $73 \mathrm{~mm}( \pm 0.05 \mathrm{~mm})$. The roll was then captured by both cameras and 3D-DIC process was performed using a grid of 441 points spaced 12 pixel $\times 12$ pixels between points $(3.35 \mathrm{~mm} \times 3.35 \mathrm{~mm})$ over a surface of 261 pixel $\times 372$ pixel. The 3 -D coordinate of points obtained in $3-\mathrm{D}$ reconstruction are shown in Fig. 5b. The first row of these $3-\mathrm{D}$ points was imported to a CAD/CAE environment to compare with an ideal circle of same dimensions. To do so, a circle of diameter $73 \mathrm{~mm}$ using both end imported points of the first row was created (Fig. 5c). The resulting reconstructed 3-D shape of the cylinder differs by an average of $0.11 \mathrm{~mm}$ (standard deviation $0.13 \mathrm{~mm}$ ) in diameter relative to the theorical cylinder. The maximum deviation between curve of 3-D points and ideal circle was $0.23 \mathrm{~mm}$ (Fig. 5c). The estimated errors are less than $1 \%$ over real measure diameter.

\subsection{Test B: related to rigid-body translating}

To determine the error related to the displacement field, a rigid body motion of the above sample was performed. To estimate this error, the cylinder was placed over caliber (positioned horizontally on plane surface). The test consists in translating the cylinder along the horizontal axes of caliber $10 \mathrm{~mm}$ and take 5 pairs of images ( 5 samples) in each position. The displacement ranges from 10 to $60 \mathrm{~mm}$. The 3DDIC parameters were the same as above test. Table 1 shows the errors, mean and SD of the errors in displacements for 5 different samples. The value of each sample corresponds to mean of variation of displacement of all 3-D points reconstructed. The estimated errors are less than $2 \%$ over the real measure $(10 \mathrm{~mm})$.

\subsection{Test C: related to out-of-plane displacement}

The out-of-plane displacement test consisted in comparing the result of a piece of aluminum with a constant load $(43.2 \mathrm{~N})$ applied to one of its extremes and its simulation in 
(a)

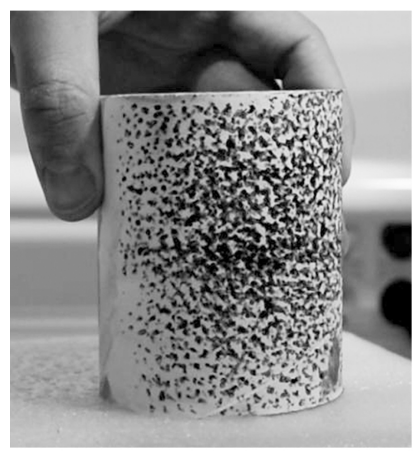

(b)

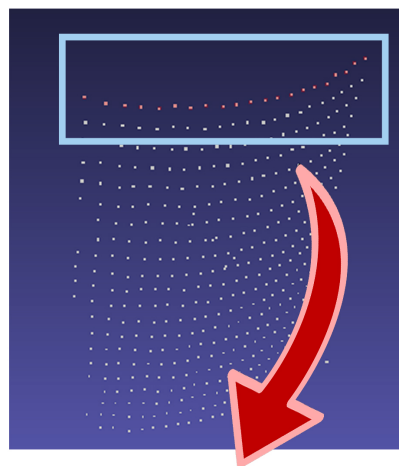

(c)

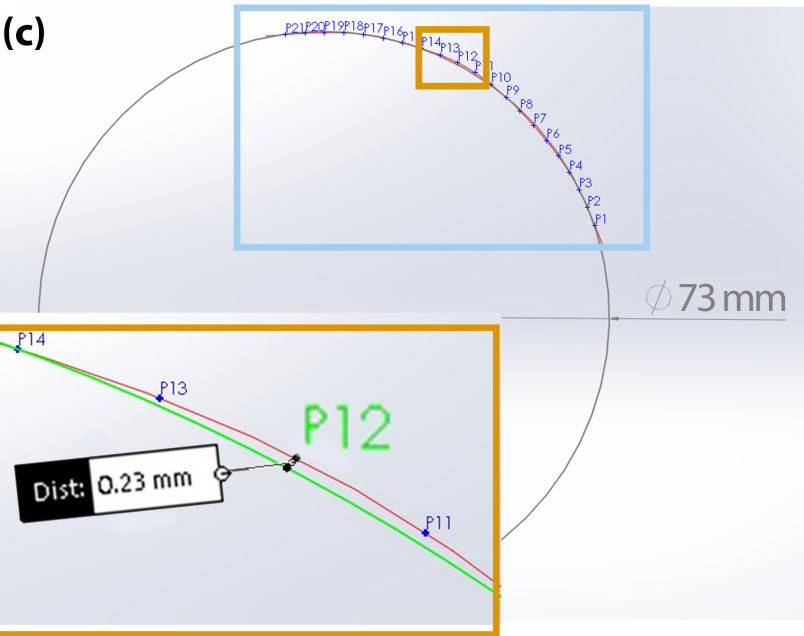

Figure 5. (a) Image of cylindrical sample with black and white speckle pattern. (b) 3-D cloud point reconstructed using 3-D stereo-system proposed. (c) Curvature check of 3-D cloud points with respect to real measured diameter of cylindrical sample in SolidWorks.

CAE tool. The piece was anchored to a structure with a external load in the other end and the images were acquired in both reference and when the external load was applied (Fig. 6a). The same situation was reconstructed in CAE tool to perform a static simulation. The results of test $\mathrm{C}$ are shown on Fig. 6. The displacements out of the plane are also calculated correctly and have approximately the same distribution and magnitude than the simulation in CAE. The maximum value of displacement was $6.361 \mathrm{~mm}$ and in the proposed method $6.5342 \mathrm{~mm}$, which represents a relative error of $2-3 \%$. Although the difference of these measures is relatively large $\left(\simeq 2 \times 10^{-2} \mathrm{~mm}\right)$, the accuracy of the method can be considered sufficient for measure large deformations suffered by human skin under muscle contractions and body segment motions which reach values of $8-10 \mathrm{~mm}$ in the foot during human gait (Blenkinsopp et al., 2012; Schmeltzpfenning et al., 2011) and even 10-15 mm in other anatomical areas such as shoulder and knee (Mahmud et al., 2010). (a)
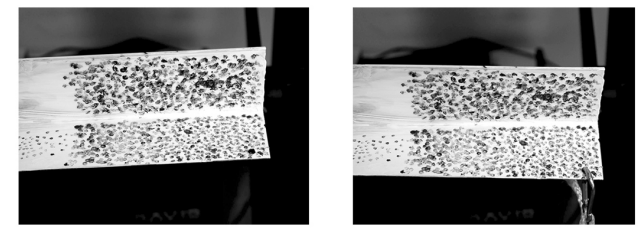

(b)

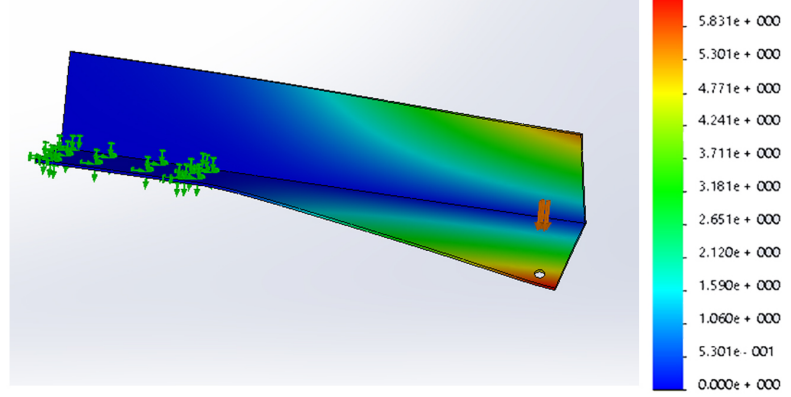

(c)
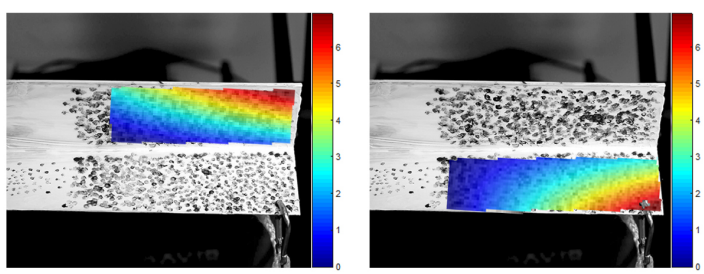

Figure 6. (a) Images of reference and deformed stage of the test piece of aluminum with the white and black speckle pattern. (b) Simulation of test in CAE enviroment. (c) Results of displacement using 3D-DIC methodology proposed.

\subsection{Test D: error in map strain}

To assess errors in strain field, a "null strain analysis" from previous test B was performed. Because the sample was subjected to a rigid body motion, any non-zero component of the tangential strain reflects an error resulting from the measurement (Fig. 7). Mean values and SD values of $\varepsilon_{x x}=$ $-4.3878 \times 10^{-4}\left( \pm 1.2145 \times 10^{-4}\right)$ and $\varepsilon_{y y}=-1.7799 \times$ $10^{-4}\left( \pm 4.951 \times 10^{-7}\right)$ were found from unfiltered strain measures. Errors in $\varepsilon_{y y}$ are three time is less than $\varepsilon_{x x}$ because the displacement in test B were in $x$-direction. Although the $\mathrm{SD}$ is aproximatelly lower that $2 \times 10^{-4}$ and $5 \times 10^{-7}$ for $\varepsilon_{x x}$ and $\varepsilon_{y y}$ respectively, the accuracy in strain field analysis can be considered sufficient for large strain fields.

\section{Results}

\subsection{Ankle-foot skin stain analysis}

Figure 8 shows the tangential and principal strains values during different stages of stance phase of human gait. The results are normalized with respect to the maximum and minimum value of the cycle. This figure shows the results from one representative repetition (one step), but the surface strain distribution showed similar patterns between steps. The strain concentration is observed at the anterior surface around the ankle in both dorsol-lateral and lateral views due 


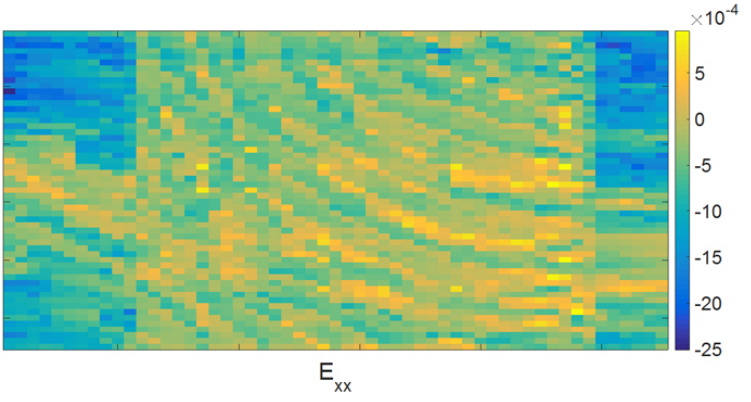

Figure 7. Errors of $\varepsilon_{x x}$ and $\varepsilon_{y y}$ obtained from the results of test B.
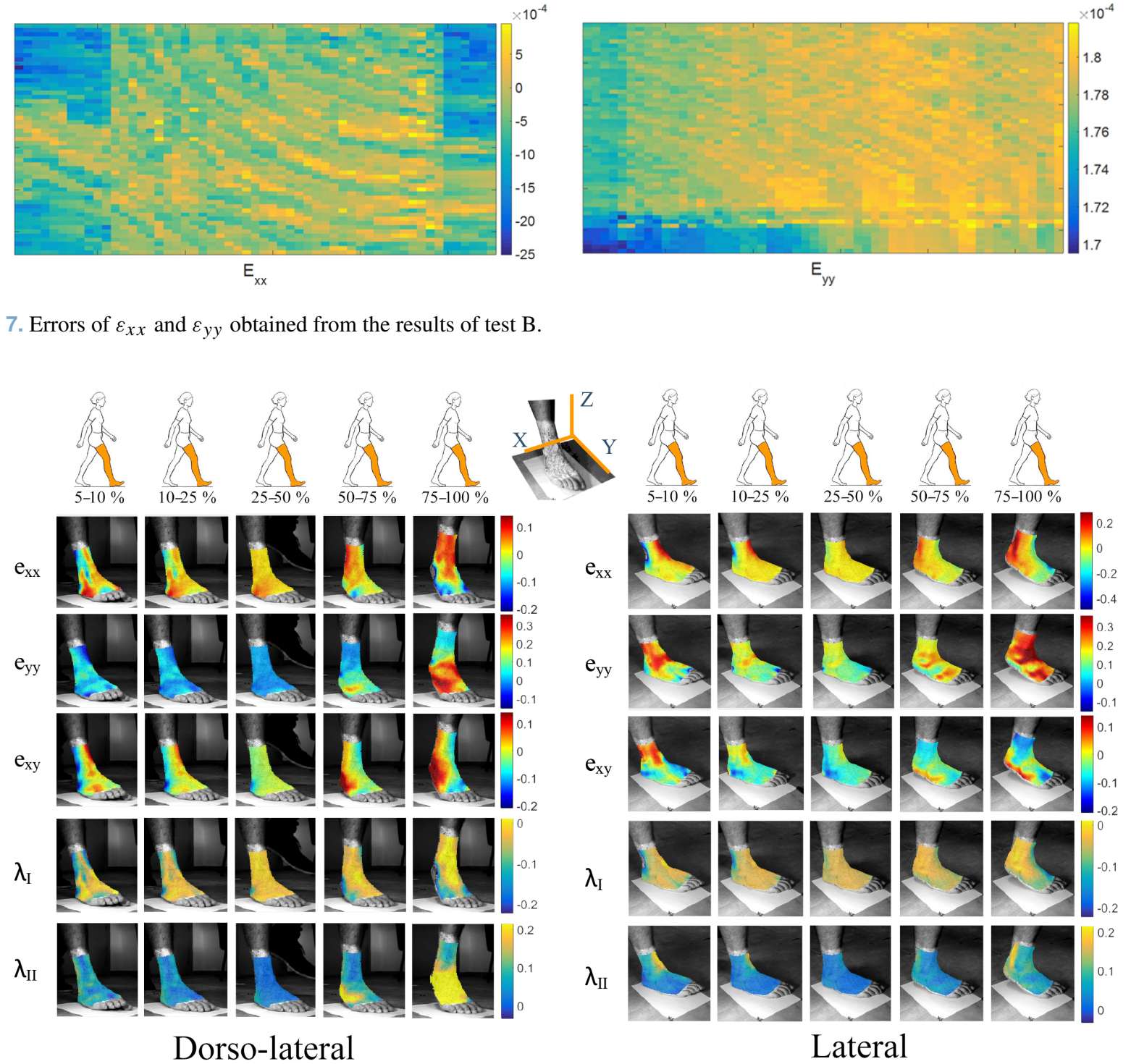

Figure 8. Skin strain field and principal strain on the dorso-lateral and lateral surface of the ankle-foot complex during walking.

to dorsiflexion of the joint. At the same time, the surface around the cuboid was contracted until the last phase stretching cycle. The principal strains in anterior and posterior surface of ankle in the early and late phases (5-25\% and 75$100 \%$ ) are dominant. In these phases of the cycle the posterior surface of ankle was contracted and stretched gradually respectively as the extension and contraction of Soleus and Gastrocnemius muscles causes traction of the Achilles tendon. By contrast, the strain magnitudes around forefoot are lower in some phases due to ausence of muscle contractions and soft tissue. This does not occur in the last phase where there is a great stretching on this area.

Mapped LoNES and directions of principal strain of the dorsi-lateral and lateral surfaces of the foot are illustrated in Fig. 9. The principal strain lines across perpendicularly areas with higher strains (anterior surface of the ankle). In the first and last phases, LoNES have higher verticality with respect the middle phases. In these middle phases, the directions of principal strain are more parallel to the direction of motion ( $Y$ axis). LoNES indicate the local directions where the deformation is minimum during motion. The LoNES vary during ankle-foot motion as shown in Fig. 9: in the initial phases (from 5 to $25 \%$ of cycle) diagonal directions of LoNES along the anterior area of ankle in both cases are shown, but in the middle phases the LoNEs are more perpendicular between them. At the same time, in the anterior surface of ankle in the first phases, the LoNES are parallel to anterior-posterior axis $(Y)$ during the motion, and in the middle phases these are parallel to the medio-lateral axis $(X)$. Moreover, in the first $(5-10 \%)$ and last phase $(75-100 \%)$ there are discontinuities 


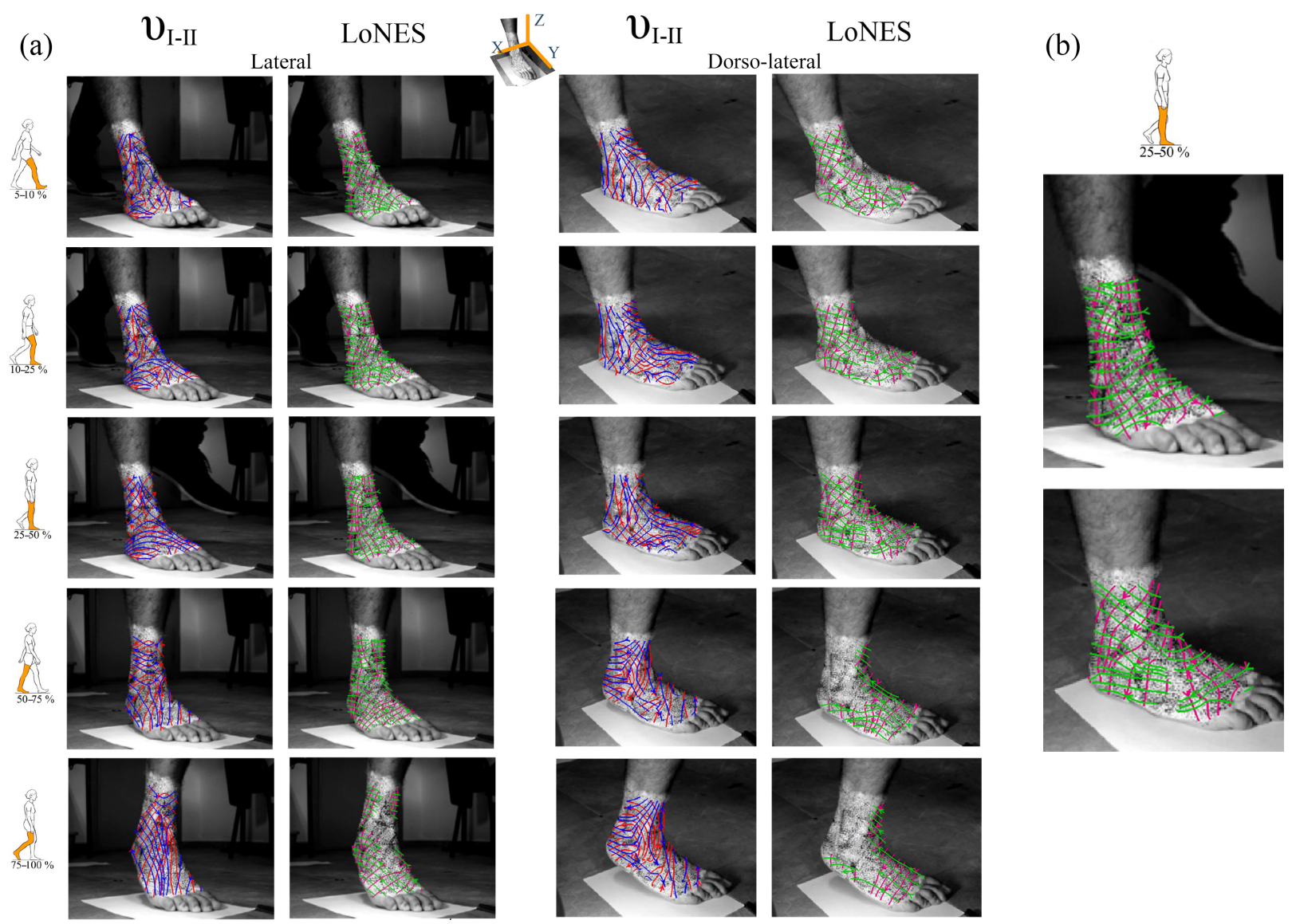

Figure 9. (a) Principal strain lines (red and blue) and LoNES (green and pink) on the dorso-lateral and lateral surface of the ankle-foot complex during walking. (b) Zoom of LoNES in middle phase (25-50\%) for a better visualization.

because LoNEs condition are not met (see Sect. 2.5), i.e., the condition $\phi-90^{\circ}>=$ is not applied. This may be due to the excessive distorsion in the skin during motion in the flexion and extension phases of the ankle joint.

Figure $9 \mathrm{~b}$ shows the LoNES in middle phase when the joint ankle is similar to anatomical position. In this phase, the LoNES are directed by the outside of the foot. In the lateral view, it is shown that LoNES avoided the malleolus area in the vertical direction. Moreover, in the calf, the LoNES are perpendicular and surround the ankle joint similar to compression bandages.

Comparison of strain results of this work with literature is challenging since methodologies, acquisition data and calculation of strain field may differ significatively between studies. However, our results are within a similar range of values and directions of LoNES reported in other works (Marreiros, 2010; Ito et al., 2016). Our methodology present a significant cost reduction compared with motion capture and 3D-DIC commecial systems used in the others studies. In this work, two different views (lateral and dorso-lateral) of ankle-foot complex to analysis the skin strain has been used.
Measuring the dynamic surface of skin using stationary camera is difficult. When a large number of camera is not available, significant effort goes into determining camera placement to best image the regions of interest. With this in mid, it is difficult to capture the entire surface of the anklefoot complex. For this reason, we choose the lateral and dorso-lateral surface to cover major area of study.

To optimize the comfort of wearable device, its properties at the interface with the skin can be modified to accommodate the natural skin deformation pattern. The skin-device interface can be reinforced along LoNES to increase the mobility and usability while providing targeted support. Moreover, an optimized interface (in terms of resisting tension or compression) can be designed using the the direction of principal strain to reduce the relative motion of body segment and decrease the shear forces between the skin and the device.

\section{Conclusions}

3D-DIC is an attractive technique that permits measure displacement and strain field of any 3-D surface in different applications. However, its setting-up needs special attention be- 
cause depends on configuration parameters which may introduce errors in the measurements. In this paper, it has proven its application to human skin strain analysis during gait using a non-commercial system developed for this purpose. The methodology is versatile and can be used for a large scale of experimental mechanics problems using conventional or high-speed cameras. The errors of displacement and strain measurement related to 3-D reconstruction and out-of-plane motion were investigated. The magnitude of these errors are acceptable in the case of large deformation (Genovese et al., 2011). The methodology presented in this work offers many advantages into area of biomechanics. Understanding the skin strain may be critical to the design of wearable devices and to optimize the comfort and usability. The data of LoNES could potentially be used for design orthosis and prosthetic liners. Thus, we expect that this low-cost methodology may serve to design the human-device interface in different applications such as exoskeletons and ortheses/prostheses. In this sense, it is necessary to continue optimizing and including different set-ups and filtering techniques for a more robust data processing procedure. Finally, to validate and check the results present in this work, more analysis in other people with and without motor pathologies it is necessary to evaluated the variability and to know the behaviour of the LoNES among these subjects.

Data availability. All the data used in this manuscript can be obtained upon request from the corresponding author.

Competing interests. The authors declare that they have no conflict of interest.

Acknowledgements. This work was supported by the Spanish Ministry of Economy and Competitiveness under project DPI201565959-C3-3R, co-financed by the European Union through EFRD funds and Gobierno of Extremadura under project TPR010.

Edited by: Andreas Müller

Reviewed by: Petar Stupar and one anonymous referee

\section{References}

Abdel-Aziz, Y. and Karara, H. M.: Direct linear transformation into object space coordinates in close-range photogrammetry, in: Proceedings of the Symposium on Close-Range Photogrammetry, University of Illionois, Urbana-Champaign, Illionois, USA, 118, 1971.

Annaidh, A. N., Bruyère, K., Destrade, M., Gilchrist, M. D., Maurini, C., Otténio, M., and Saccomandi, G.: Automated estimation of collagen fibre dispersion in the dermis and its contribution to the anisotropic behaviour of skin, Ann. Biomed. Eng., 40, 16661678, https://doi.org/10.1007/s10439-012-0542-3, 2012a.
Annaidh, A. N., Bruyère, K., Destrade, M., Gilchrist, M. D., and Otténio, M.: Characterization of the anisotropic mechanical properties of excised human skin, J. Mech. Behav. Biomed. Mater., 5, 139-148, https://doi.org/10.1016/j.jmbbm.2011.08.016, 2012 b.

Begonia, M., Dallas, M., Johnson, M. L., and Thiagarajan, G.: Comparison of strain measurement in the mouse forearm using subject-specific finite element models, strain gaging, and digital image correlation, Biomech. Model. Mechan., 16, 1243-1253, https://doi.org/10.1007/s10237-017-0885-7, 2017.

Blenkinsopp, R., Harland, A., Price, D., Lucas, T., and Roberts, J.: A method to measure dynamic dorsal foot surface shape and deformation during linear running using digital image correlation, Procedia Engineer., 34, 266-271, https://doi.org/10.1016/j.proeng.2012.04.046, 2012.

Chu, T. C., Ranson, W. F., and Sutton, M. A.: Applications of digital-image-correlation techniques to experimental mechanics, Exp. Mech., 25, 232-244, https://doi.org/10.1007/BF02325092, 1985.

Domingues, A. R., Marreiros, S. P., Martins, J. M., Silva, M. T., and Newman, D. J.: Skin Strain Field Analysis of the Human Ankle Joint, in: $4^{\circ}$ Congresso Nacional de Biomecânica (CNB2011), Coimbra, Portugal, 2011.

Elguedj, T., Réthoré, J., and Buteri, A.: Isogeometric analysis for strain field measurements, Comput. Method. Appl. M., 200, 4056, https://doi.org/10.1016/j.cma.2010.07.012, 2011.

Evans, S. L. and Holt, C. A.: Measuring the mechanical properties of human skin in vivo using digital image correlation and finite element modelling, J. Strain Anal. Eng., 44, 337-345, https://doi.org/10.1243/03093247JSA488, 2009.

Geerligs, M., Oomens, C., Ackermans, P., Baaijens, F., and Peters, G.: Linear shear response of the upper skin layers, J. Biorheol., 48, 229-245, https://doi.org/10.3233/BIR-2011-0590 2011a.

Geerligs, M., Van Breemen, L., Peters, G., Ackermans, P., Baaijens, F., and Oomens, C.: In vitro indentation to determine the mechanical properties of epidermis, J. Biomech., 44, 1176-1181, https://doi.org/10.1016/j.jbiomech.2011.01.015, 2011 b.

Genovese, K., Lee, Y. U., and Humphrey, J. D.: Novel optical system for in vitro quantification of full surface strain fields in small arteries: I. Theory and design, Comput. Method. Biomec., 14, 213-225, https://doi.org/10.1080/10255842.2010.545823, 2011.

Gu, X., Liang, Y., and Fu, S.: Evaluation and optimization of digital image correlation processing variables using genetic algorithm, J. Strain Anal. Eng., 51, 408-415, https://doi.org/10.1177/0309324716645704, 2016.

Hatze, H.: High-precision three-dimensional photogrammetric calibration and object space reconstruction using a modified DLTapproach, J. Biomech., 21, 533-538, 1988.

Hendriks, F. M., Brokken, D., Van Eemeren, J. T. W. M., Oomens, C. W. J., Baaijens, F. P. T., and Horsten, J. B. A. M.: A numericalexperimental method to characterize the non-linear mechanical behaviour of human skin, Skin Res. Technol., 9, 274-283, https://doi.org/10.1016/j.medengphy.2005.07.001, 2003.

Hendriks, F. M., Brokken, D., Oomens, C. W. J., Bader, D. L., and Baaijens, F. P. T.: The relative contributions of different skin layers to the mechanical behavior of human skin in vivo using suction experiments, Med. Eng. Phys., 28, 259-266, 2006.

Iberall, A. S.: The Use of Lines of Nonextension to Improve Mobility in Full-Pressure Suits. AMRL-TR-64-118., AMRL-TR Aerospace Medical Research Laboratories (6570th), p. 1, 1964. 
Ito, K., Maeda, K., Fujiwara, I., Hosoda, K., Nagura, T., Lee, T., and Ogihara, N.: Dynamic measurement of surface strain distribution on the foot during walking, J. Mech. Behav. Biomed., 69, 249256, https://doi.org/10.1016/j.jmbbm.2016.12.009, 2016.

Kimura, M., Mochimaru, M., and Kanade, T.: Measurement of 3D foot shape deformation in motion, in: Proceedings of the 5th ACM/IEEE International Workshop on Projector camera systems, ACM, New York, USA, 10:1-10:8, https://doi.org/10.1145/1394622.1394636, 2008.

Kwiatkowska, M., Franklin, S., Hendriks, C., and Kwiatkowski, K.: Friction and deformation behaviour of human skin, Wear, 267, 1264-1273, 2009.

Lin, B., Moerman, K. M., McMahan, C. G., Pasch, K. A., and Herr, H. M.: Low-Cost Methodology for Skin Strain Measurement of a Flexed Biological Limb, IEEE T. Bio-Med. Eng., PP, 3564-3567, https://doi.org/10.1109/TBME.2016.2626442, 2016.

Luo, P. F., Chao, Y. J., Sutton, M. A., and Peters, W.-H.: Accurate measurement of three-dimensional deformations in deformable and rigid bodies using computer vision, Exp. Mech., 33, 123132, https://doi.org/10.1007/BF02322488, 1993.

Mahmud, J., Holt, C. A., and Evans, S. L.: An innovative application of a small-scale motion analysis technique to quantify human skin deformation in vivo, J. Biomech., 43, 1002-1006, https://doi.org/10.1016/j.jbiomech.2009.11.009, 2010.

Marreiros, S. S. P.: Skin Strain Field Analysis of the Human Ankle Joint, Relation, 2, 2-7, 2010.

Miura, N., Arikawa, S., Yoneyama, S., Koike, M., Murakami, M., and Tanno, O.: Digital image correlation strain analysis for the study of wrinkle formation on facial skin, Journal of Solid Mechanics and Materials Engineering, 6, 545-554, https://doi.org/10.1299/jmmp.6.545, 2012.

Moerman, K. M., Holt, C. A., Evans, S. L., and Simms, C. K.: Digital image correlation and finite element modelling as a method to determine mechanical properties of human soft tissue in vivo, J. Biomech., 42, 1150-1153, https://doi.org/10.1016/j.jbiomech.2009.02.016, 2009.

Newman, D., Hoffman, J., Bethke, K., Blaya, J., Carr, C., and Pitts, B.: Astronaut bio-suit system for exploration class missions, NIAC Phase II Final Report, 2005.

Obropta, E. W. and Newman, D. J.: Skin strain fields at the shoulder joint for mechanical counter pressure space suit development, in: 2016 IEEE Aerospace Conference, Big Sky, MT, USA, 1-9, https://doi.org/10.1109/AERO.2016.7500744, 2016.

Obropta Jr., E. W.: On the deformation of human skin for mechanical counter pressure space suit development, $\mathrm{PhD}$ thesis, Massachusetts Institute of Technology, 2015.

Pan, B. and Li, K.: A fast digital image correlation method for deformation measurement, Opt. Laser. Eng., 49, 841-847, https://doi.org/10.1016/j.optlaseng.2011.02.023, 2011.

Pan, B., Xie, H., Guo, Z., and Hua, T.: Full-field strain measurement using a two-dimensional Savitzky-Golay digital differentiator in digital image correlation, Opt. Eng., 46, 033601, https://doi.org/10.1117/1.2714926, 2007.

Pan, B., Xie, H., and Wang, Z.: Equivalence of digital image correlation criteria for pattern matching, Appl. Opt., 49, 5501-5509, https://doi.org/10.1364/AO.49.005501, 2010.

Quan, C., Tay, C. J., Sun, W., and He, X.: Determination of three-dimensional displacement using two-dimensional digital image correlation, Appl. Optics, 47, 583-593, https://doi.org/10.1364/AO.47.000583, 2008.

Réthoré, J., Hild, F., and Roux, S.: Shear-band capturing using a multiscale extended digital image correlation technique, Comput. Method. Appl. M., 196, 5016-5030, https://doi.org/10.1016/j.cma.2007.06.019, 2007.

Schmeltzpfenning, T., Plank, C., Fritz, B., Aswendt, P., and Grau, S.: 3D dynamic behaviour of foot structure may provide additional information for last design, Footwear Science, 3, S147S148, https://doi.org/10.1080/19424280.2011.575866, 2011.

Seo, H., Kim, S.-j., Cordier, F., Choi, J., and Hong, K.: Estimating dynamic skin tension lines in vivo using 3D scans, Comput.-Aided Des., 45, 551-555, https://doi.org/10.1016/j.cad.2012.10.044, 2013.

Staloff, I. A., Guan, E., Katz, S., Rafailovitch, M., Sokolov, A., and Sokolov, S.: An in vivo study of the mechanical properties of facial skin and influence of aging using digital image speckle correlation, Skin Res. Technol., 14, 127-134, https://doi.org/10.1111/j.1600-0846.2007.00266.x, 2008.

Tang, Z., Liang, J., Xiao, Z., and Guo, C.: Large deformation measurement scheme for 3D digital image correlation method, Opt. Laser Eng., 50, 122-130, https://doi.org/10.1016/j.optlaseng.2011.09.018, 2012.

Tang, Z.-Z., Liang, J., Xiao, Z.-Z., Guo, C., and Hu, H.: Threedimensional digital image correlation system for deformation measurement in experimental mechanics, Opt. Eng., 49, 103601, https://doi.org/10.1117/1.3491204, 2010.

Thabet, A. K., Trucco, E., Salvi, J., Wang, W., and Abboud, R. J.: Dynamic 3D shape of the plantar surface of the foot using coded structured light: a technical report, J. Foot Ankle Res., 7, 1-12, https://doi.org/10.1186/1757-1146-7-5, 2014.

Tran, H. V., Charleux, F., Rachik, M., Ehrlacher, A., and Ho Ba Tho, M. C.: In vivo characterization of the mechanical properties of human skin derived from MRI and indentation techniques, Comput. Method. Biomec., 10, 401-407, https://doi.org/10.1080/10255840701550287, 2007.

Van den Herrewegen, I., Cuppens, K., Broeckx, M., BarischFritz, B., Vander Sloten, J., Leardini, A., and Peeraer, L.: Dynamic 3D scanning as a markerless method to calculate multi-segment foot kinematics during stance phase: Methodology and first application, J. Biomech., 47, 2531-2539, https://doi.org/10.1016/j.jbiomech.2014.06.010, 2014.

Vilhena, L. and Ramalho, A.: Friction of human skin against different fabrics for medical use, Lubricants, 4, 6, https://doi.org/10.3390/lubricants4010006, 2016.

Wehner, M., Quinlivan, B., Aubin, P. M., Martinez-Villalpando, E., Baumann, M., Stirling, L., Holt, K., Wood, R., and Walsh, C.: A lightweight soft exosuit for gait assistance, in: 2013 IEEE International Conference on Robotics and Automation (ICRA), IEEE, 3362-3369, https://doi.org/10.1109/ICRA.2013.6631046, 2013.

Yoneyama, S.: Computing strain distributions from measured displacements on a three-dimensional surface, The Japan Society of Mechanical Engineers, 10, s113-s118, https://doi.org/10.11395/jjsem.10.s113, 2010.

Yoneyama, S.: Smoothing measured displacements and computing strains utilising finite element method, Strain, 47, 258-266, https://doi.org/10.1111/j.1475-1305.2010.00765.x, 2011. 